\title{
Leaching and kinetics process of cobalt from used lithium ion batteries with organic citric acid
}

\author{
Yuliusman ${ }^{1, *}$, Annisaa Nurqomariah $^{1}$, Radifan Fajaryanto ${ }^{1}$, Silvia ${ }^{1}$ \\ Department of Chemical Engineering, Universitas Indonesia, Depok 16424, Indonesia
}

\begin{abstract}
Batteries waste is found anywhere in the world because most of the electronic devices use batteries to operate them. Batteries that were used for electronic devices nowadays is lithium ion batteries. Lithium ion batteries is one of the rechargeable batteries where it contained heavy metals which is very dangerous for health and environment. However, in order to minimalize the composition before its being disposed many researchers have found a way to recover heavy metals, because it can be useful to reproduce lithium ion batteries in the future. In this research, hydrometallurgical leaching process has been done for recovery cobalt from used lithium ion using hydrogen peroxide as reducing agent and citric acid as a leaching agent by varying citric acid concentration $(0-2 \mathrm{M})$, reaction temperature $\left(50-80^{\circ} \mathrm{C}\right)$, and reaction time $(5-60$ minutes). The result were analyzed by atomic absorption spectroscopy (AAS) and showed that the best operation condition is at concentration $1 \mathrm{M}$, temperature $80^{\circ} \mathrm{C}$ with stirring time 60 minutes can recover more than $90 \%$ of Cobalt.
\end{abstract}

\section{Introduction}

Today, the technology industry has grown rapidly which implies to the massive production and used of lithium ion batteries. Lithium ion batteries were used on most electronic device such as mobile phones and laptops. Lithium ion batteries were chosen because of its long storage life. However, lithium ion batteries were considered as waste management and it is very dangerous for health and environment, because it contain heavy metals such as lithium, cobalt, nickel and copper [1].

Lithium ion batteries contain recoverable heavy metals which are Cobalt, Lithium and Nickel. The composition of lithium ion batteries include 5-20\% cobalt, $5-10 \%$ nickel, $5-7 \%$ lithium, $15 \%$ organic chemicals, and 7\% plastics [2]. Many researchers have found a way to minimalize heavy metals composition before its being disposed by recovering. Looking at its composition, it can be concluded that composition of Cobalt in lithium ion batteries is high and can be recovered.

To recover heavy metals, various methods were investigated, such as pyrometallurgy and hydrometallurgy. In order to obtain high recovery percentage with small energy consumption hydrometallurgical process was chosen. Hydrometallurgical process has two main stages, pretreatment and main treatment.

Pre-treatment includes disassembling, destruction and filtering batteries. While the main treatment includes leaching, chemical extraction and precipitation. Hydrometallurgy is considered to be the best processes in recovering metals from battery waste, especially used lithium ion, because lithium ion batteries are easily leached [3].

Leaching is a solid-liquid extraction method where the separation process between metals inside the batteries and leaching agent. Used lithium ion batteries will be mixed with leaching agent and allow the metal inside the solid to dissolve in the leaching agent. Leaching agents commonly used are inorganic acids such as $\mathrm{HCL}, \mathrm{HNO}_{3}$, and $\mathrm{H}_{2} \mathrm{SO} 4$ [4]. However, the use of strong acids as leaching agent is harmful to the environment because it produce gases emissions like $\mathrm{Cl}_{2}$ and $\mathrm{SO}_{3}$. Those gas can harm the ecosystem and our health.

To solve this problem, researchers began using organic acids as leaching agents. The reason why researcher began to substitute inorganic acid to organic acid is because the waste from the process is easier to process [5]. Moreover it is also environmentally friendly and their characteristics are easily degraded by natural. The only disadvantage of using organic acid as leaching agent is its recovery percentage, because most of organic acid is weak acid, so organic acid tend to recover less than inorganic acid. Organic acid used as a leaching agents are citric acid, ascorbic acid, and DL-malic.

In previous study, citric acid $\left(\mathrm{C}_{6} \mathrm{H}_{8} \mathrm{O}_{7}\right)$ and hydrogen peroxide $\left(\mathrm{H}_{2} \mathrm{O}_{2}\right)$ were once used as leaching agents and reducing agents were able to recover $92.53 \%$ Co [6]. Another experiment using organic acid as leaching agent and hydrogen peroxide $\left(\mathrm{H}_{2} \mathrm{O}_{2}\right)$ as reducing agent can recover $93 \%$ [7]. Based on the above data, it is known that organic acid is now widely used. Generally during the leaching process, reducing agent were added in order to increase its recovery percentage [3]. Researcher found that the presence of reducing agent can accelerate the

Corresponding author: usman@che.ui.ac.id 
recovery efficiency. Recent research found that hydrogen peroxide was the most efficient reducing agent among glucose and ascorbic acid [8].

\section{Materials and method}

\subsection{Materials and reagents}

Used lithium-ion batteries from mobile phone were collected from the local market. In this research, citric acid is used as leaching agent and hydrogen peroxide is used as reducing agent. Solutions were prepared using distilled water.

\subsection{Dismantling and separation lithium ion batteries}

Used lithium-ion batteries were soaked with $1 \mathrm{wt} \% \mathrm{NaCl}$ solution for 24 hour to discharge batteries process, because it might produce fire during the dismantling. After discharging, used lithium ion batteries were dismantled and were separated from its main components which are casing, anode and cathode. This research will use cathode component, because it contains $\mathrm{LiCoO}_{2}$. The cathode is a black powder which attached to the aluminium foil. Cathode was taken using spatula and were grounded until it became a fine powder. After it became fine powder, the powder were sieved to make a uniform particle size (100 mesh).

\subsection{Leaching procedure}

Leaching process were carried out in $250 \mathrm{ml}$ pyrex glass reactor which was placed over hot plate with magnetic stirrer with controlled stirring speed and temperature. To control the temperature during process, pyrex glass was equipped with thermometer and were closed using alumunium foil to avoid the evaporation of the solution. Citric acid was first poured into the reactor along with the hydrogen peroxide and allowed to reach the thermal equilibrium. A known amount of cathode powder were later poured into the reactor after its equilibrium and stirred with magnetic stirrer. During the variation of citric acid concentration and temperature reactor was stirred for 60 minutes. Solid/liquid ration $(20 \mathrm{~g} / \mathrm{L})$ with known stirring speed and also $2 \%$ of hydrogen peroxide. Citric acid concentration $(0-2 \mathrm{M})$, reaction temperature $\left(50-80^{\circ} \mathrm{C}\right)$, reaction time $(5-60$ minutes) were varied in this leaching process. Reaction time were varied in order to achieve kinetics studies. After leaching process, the solution were filtrate and analyzed using AAS (Shimadzu AA-6300) to determine its cobalt content.

\section{Results and discussion}

\subsection{Effect of citric acid concentration}

Effect of varied citric acid concentration $(0 \mathrm{M}, 0.5 \mathrm{M}, 1$ $\mathrm{M}, 1.5 \mathrm{M}$ and $2 \mathrm{M}$ ) has been done with solid/liquid ration $(20 \mathrm{~g} / \mathrm{L})$, reaction temperature $80^{\circ} \mathrm{C}$, reaction time 60 minutes and $2 \%$ of hydrogen peroxide. This step is aim to determine the effect of leaching reaction by varying leaching agent concentration. Fig. 1 shows the effect of citric acid concentration on leaching Cobalt.

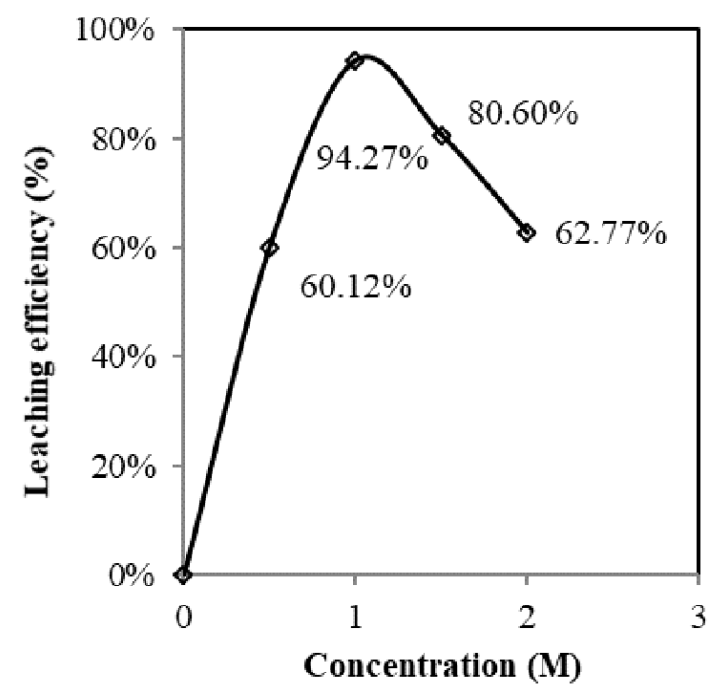

Fig. 1. Effect of citric acid concentration on leaching Cobalt.

Based on Fig. 1 it shows that the increasing citric acid concentration will increase leaching efficiency. The increasing of concentration will increase the activity of $\mathrm{H}^{+}$and more proton will bound with metal ions [9]. Basically the molecule structure of citric acid consist of carboxyl group (-COOH), $\mathrm{H}^{+}$in carboxyl will bind to dissolved metals ions. The more carboxyl group acid has, the more metal ions can be dissolved in the solution. Compared to the other organic acid, citric acid has the most carboxyl acid, which is good for leaching process [6]. The dissociation of citric acid can be shown in eq. $(1-3)$ :

$$
\begin{aligned}
& \mathrm{H}_{3} \mathrm{C}_{8} \mathrm{H}_{5} \mathrm{O}_{7} \leftrightarrow \mathrm{H}_{2} \mathrm{C}_{8} \mathrm{H}_{5} \mathrm{O}_{7}^{-}+\mathrm{H}^{+} \\
& \mathrm{H}_{2} \mathrm{C}_{8} \mathrm{H}_{5} \mathrm{O}_{7}^{-} \leftrightarrow \mathrm{HC}_{8} \mathrm{H}_{5} \mathrm{O}_{7}^{2-}+\mathrm{H}^{+} \\
& \mathrm{HC}_{8} \mathrm{H}_{5} \mathrm{O}^{2-} \leftrightarrow \mathrm{C}_{8} \mathrm{H}_{5} \mathrm{O}_{7}{ }^{3-}+\mathrm{H}^{+}
\end{aligned}
$$

The leaching efficiency slowly decreasing because of citric acid is a weak acid and weak acid cannot be fully ionized. The ability and amount of anion formation of weak electrolytes tend to follow the graph trend until its optimum condition. The relationship between weak electrolyte and its conductivity, whereas the concentration increase, the conductivity will increase to a certain extent. If it is increased further, it will decrease the conductivity and also will decrease the leaching efficiency [10]. The optimum acid citric concentration is $1 \mathrm{M}$. 


\subsection{Effect of reaction temperature}

Effect of varied reaction temperature $\left(50^{\circ} \mathrm{C}, 60^{\circ} \mathrm{C}, 70^{\circ} \mathrm{C}\right.$, and $80^{\circ} \mathrm{C}$ ) has been done with $1 \mathrm{M}$ citric acid, solid/liquid ratio $(20 \mathrm{~g} / \mathrm{L})$, reaction temperature $80^{\circ} \mathrm{C}$, reaction time 60 minutes and $2 \%$ of hydrogen peroxide. This step aim to determine the effect of leaching reaction by varying reaction temperature. Fig. 2 shows the effect of reaction temperature on leaching Cobalt. According to the graph below it can be concluded that the percentage of leaching will increase as the reaction temperature increases. Increasing leaching efficiency is due to the dissociation of citric acid present in endothermic reaction. If it is compared to strong acids, citric acid will require high temperature to achieve high percentage of leaching efficiency, because high temperature will result more $\mathrm{H}^{+}$to disconnect from citric acid. However, when the operating temperature is above $90^{\circ} \mathrm{C}$, leaching efficiency will decrease because citric acid will evaporate from the solution [7]. The operating temperature also affects the rate of chemical reaction and ion transfer rate [7] and effect the activation energy. The optimum reaction temperature result is $80^{\circ} \mathrm{C}$.

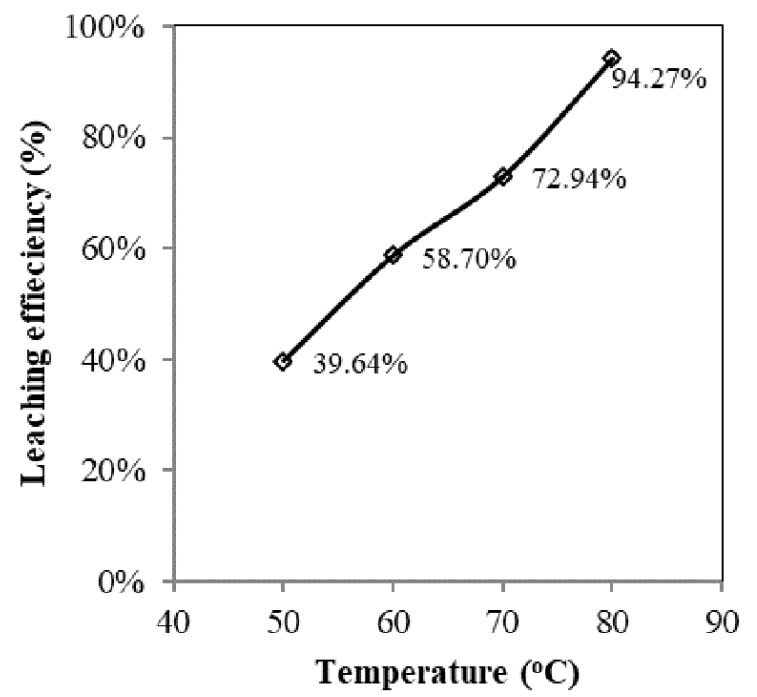

Fig. 2 Effect of reaction temperature on leaching Cobalt.

\subsection{Kinetics study}

The kinetic study was conducted to determine the mechanism of leaching reaction, because leaching lithium battery is include in the heterogeneous reaction, where the process can be controlled by chemical reaction or diffusion. The heterogeneous reaction controlled by the diffusion is dependent to the operating temperature, whereas if the process is controlled by a chemical reaction, the process is highly dependent on the operating temperature. With this study it will determine whether this process will be controlled by chemical reaction or diffusion.

The kinetic study is performed by varying stirring time and temperature of leaching reaction. The leaching process was performed with solid/liquid ratio of 20 $\mathrm{mg} / \mathrm{L}$, citric acid concentration $1 \mathrm{M}$, and $2 \%$ of hydrogen peroxide. The temperature were varied from $50^{\circ} \mathrm{C}, 60^{\circ} \mathrm{C}$, $70^{\circ} \mathrm{C}$, and $80^{\circ} \mathrm{C}$. The stirring time was varied from 5 minutes, 10 minutes, 15 minutes, 30 minutes, and 60 minutes. Fig.3 shows the effect of stirring time on leaching Cobalt. From the graph below, it can be concluded that higher reaction temperature will result higher leaching efficiency. The temperature is linear to reaction time, longer reaction time will result higher leaching efficiency. But at certain point leaching efficiency will not be significant. This is due to $\mathrm{H}^{+}$ activities that are difficult to dissociate as they reach their saturation point and resulting constant percentage of leaching.

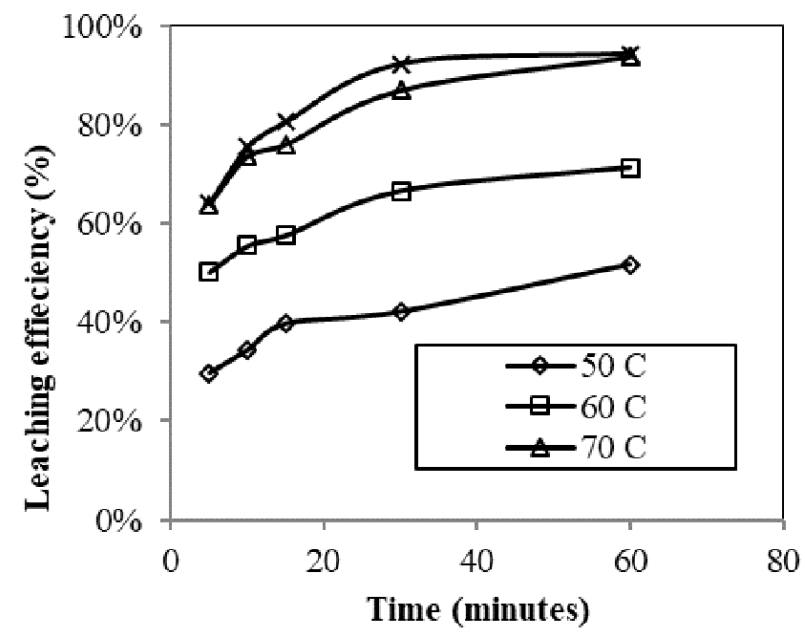

Fig. 3 Effect of stirring time on leaching Cobalt

To determine the mechanism of the leaching process evaluate, it will require a test that fit with the shrinking core model. The kinetic data will be obtain from leaching experiments at different temperatures. There are two kinetic models are presented explained in eq. (4-5). Eq. (4) is when the reaction is chemically controlled at the surface, while Eq. (5) is when reaction is controlled by diffusion through product layer [11].

$$
\begin{gathered}
1-\frac{2}{a} X-(1-X)^{2}=k_{d} t \\
1-(1-X)^{\frac{1}{a}}=k_{c} t
\end{gathered}
$$

Where $\mathrm{x}$ is the fraction reacted in time $(\mathrm{t}), \mathrm{k}_{\mathrm{c}}$ is chemical reaction rate constant $\left(\mathrm{min}^{-1}\right)$ and $\mathrm{k}_{\mathrm{d}}$ is apparent diffusion reaction rate constant $\left(\mathrm{min}^{-1}\right)$. 


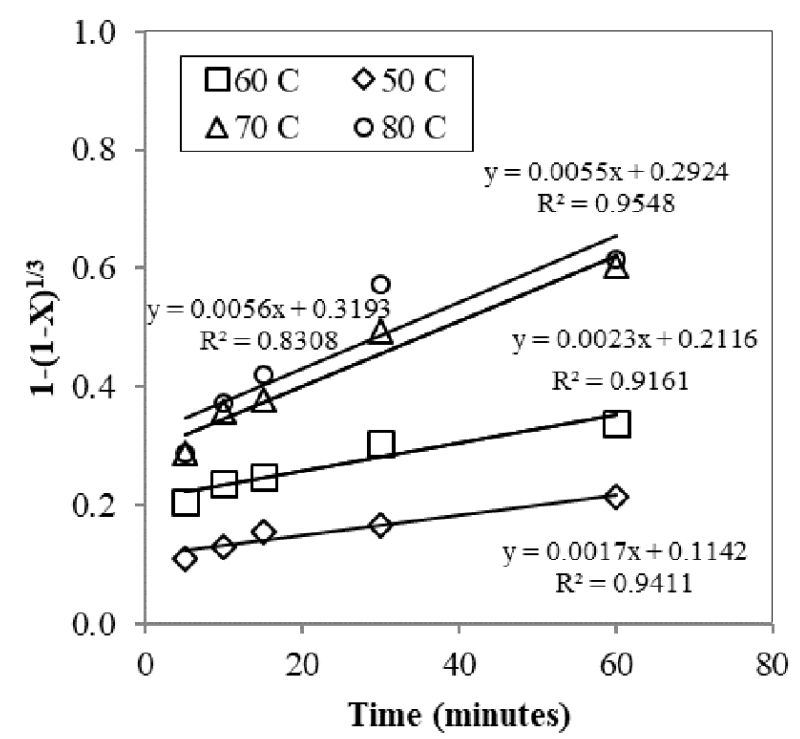

Fig. 4 Plot of $1-(1-X)^{1 / 3}$ vs t (minutes) for Cobalt

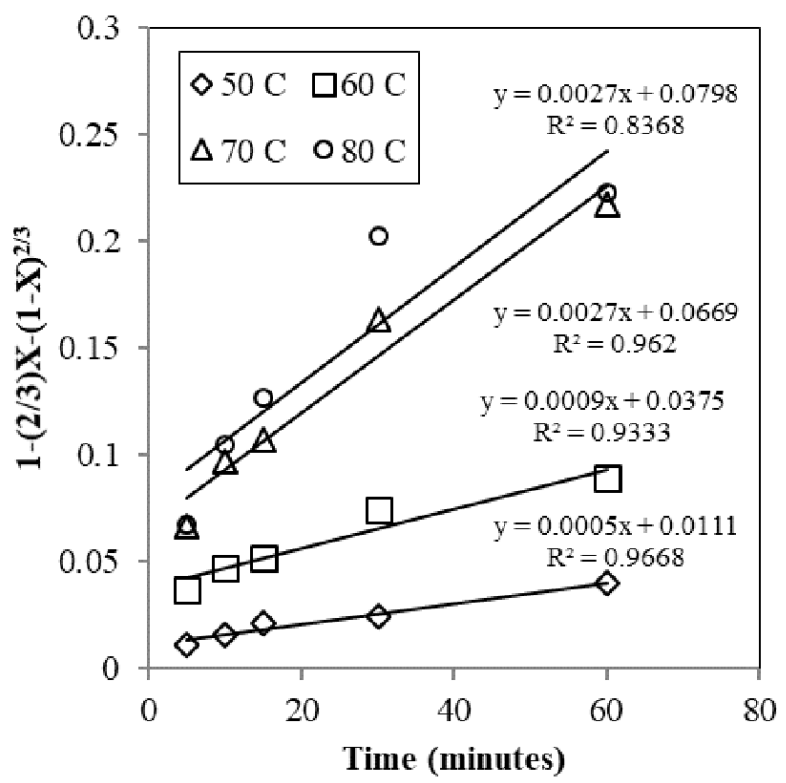

Fig. $51-2 / 3 X-(1-X)^{2 / 3}$ vs $t$ for Cobalt.

The leaching efficiency will be plotted using eq. (4-5) with leaching time. This method is aim to make it easier to choose the suitable model for this research. The suitable model was seen from the value of correlation coefficient that approach to 1 .

\subsection{Energy Activation}

Energy activation is the energy needed for to start the reaction, or energy that must be absorbed by the system from the environment to initiate reaction. Based on the result of kinetic studies, it can be used to calculate the energy activation. Energy activation can be calculate using Arrhenius eq. (6-7)

$$
k=A e^{\frac{-k T H}{K T}}
$$

$$
\operatorname{Ln}(k)=\operatorname{Ln}(A)-\frac{E a}{T} \cdot \frac{1}{T}
$$

Where $\mathrm{A}$ is pre-exponential factor $(1 / \mathrm{min}), \mathrm{Ea}$ is activation energy $(\mathrm{J} / \mathrm{mole}), \mathrm{R}$ is universal gas constant $(\mathrm{J} /$ mole. $\mathrm{K}), \mathrm{T}$ is temperature $(\mathrm{K})$ and $\mathrm{k}$ is the reaction rate constant. To obtain the energy activation of Cobalt, $\ln (\mathrm{k})$ value is obtained from constant in the surface chemical reaction control model graph. By plotting $\ln$ $(\mathrm{K})$ and $1 / \mathrm{T}$, energy activation can be calculated. Fig 5. Shows the Arrhenius plot of leaching Cobalt (ln (k) vs $1 / \mathrm{T})$. The slope is the value of $-\frac{E_{a}}{R}$, therefore energy activation can be calculate. Energy activation can be calculate by multiplying the gradient with common gas constant $(\mathrm{kJ} / \mathrm{K}$. mole). Activation energy obtained for chemical reaction model of $42.2975 \mathrm{~kJ} / \mathrm{mol}$. From recent studies, if leaching process is controlled by diffusion, the activation energy will be less than $40 \mathrm{~kJ} / \mathrm{mol}$, but if leaching process is controlled by chemical reaction, the activation energy will be more than $40 \mathrm{~kJ} / \mathrm{mol}$. Therefore, it can be concluded that leaching process with organic citric acid is controlled by chemical reaction.

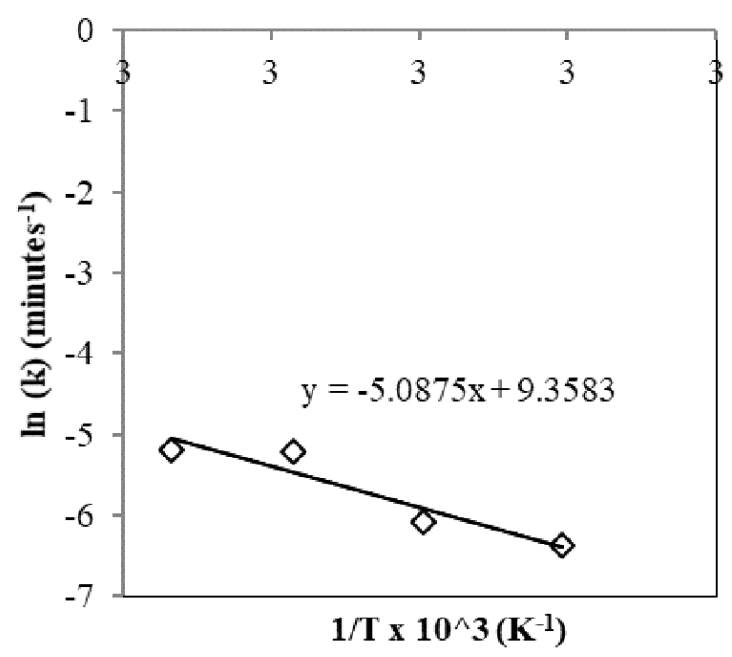

Fig. 5 Arrhenius plot of Leaching Cobalt

\section{Conclusions}

Based on this experiment, leaching efficiency can be increased with many factors which include citric acid concentration, reaction temperature and reaction time. This study also showed that the best operation condition is at concentration $1 \mathrm{M}$, temperature $80^{\circ} \mathrm{C}$ with stirring time 60 minutes can recover more than $90 \%$ of Co. Based on kinetics study, leaching reaction of cobalt using citric acid is controlled by surface chemical reaction model with the activation energy of 42.29 $\mathrm{kJ} / \mathrm{mole}$.

\section{References}

1. D. H. P. Kang, M. Chen, \& O. A. Orgunseitan. Potential Environmental and Human Health 
Impacts of Rechargeable Lithium Batteries in Electronic Waste. Environmental Science \& Technology, 47(10), 5495-5503 (2013)

2. S. M. Shin, N. H. Kim, J. S Sohn, D.H Yang. Y.H. Kim. Development of a Metal Recovery Process from Li-Ion Battery Wastes. Hydrometallurgy, 79, 172-181 (2005)

3. Q. Wang, B. Jiang, B. Li, \& Y. Yan. A Critical Review of Thermal Management Models and Solutions of Lithium-Ion Batteries for the Development of Pure Electric Vehicle. Renewable and Sustainable Energy Reviews, 64, 106-128 (2016)

4. S. Peelman, Z. H. I Sun, J. Sietsma, \& Y. Yang. Leaching of Rare Earth Elements: Past and Present. ERES2014: 1st European Rare Earth Resources Conference, 446-456 (2014)

5. L. Li, J. B. Dunn, X. X. Zhang, L. Graines, R. J. Chen, F. Wu, \& K. Amine. Recovery of Metals from Spent Lithium-Ion Batteries with Organic Acids as Leaching Reagents and Environmental Assessment. Journal of Power Sources, 233, 180-189 (2013)

6. R, Golmohammadzade, F. Rashchi, \& E. Vahidi. Recovery of Lithium and Cobalt from Spent Lithium-Ion Batteries using Organic Acids : Process Optimization and Kinetic Aspects, 64, 244-254 (2017)

7. L. Li, J. Ge, R. Chen, F. Wu, S. Chen \& X. Zhang. Environmental Friendly Leaching Reagent for Cobalt and Lithium Recovery from Spent Lithium-Ion Batteries, 30, 2615-2621 (2010)

8. M. Aaltonen, C. Peng, B. Wilson, \& M. Lundström. Leaching of Metals from Spent Lithium-Ion Batteries. Recycling, 2(4), 20 (2017)

9. J. S. Guzolu, M. Gharabaghi, M. Mobin, \& H. Alilo. Extraction of $\mathrm{Li}$ and $\mathrm{Co}$ from Li-ion Batteries by Chemical Methods. Journal of The Institution of Engineers (India): Series D. (2016).

10. Lower, Stephen. Chem1 General Chemistry Virtual Textbook. Canada: Simon Fraser University (2016)

11. P. Meshram, B. D. Pandey, \& T. R. Mankhand. Hydrometallurgical Processing of Spent Lithium Ion Batteries ( LIBs ) in the Presence of a Reducing Agent with Emphasis on Kinetics of Leaching. Chemical Engineering Journal, 281, 418-427 (2016). 